\title{
Erratum zu: Der Aufbau einer Organisationsrichtlinie für den Daten- und Informationsqualitätsmanagement-Prozess
}

\section{Erratum zu: K. Hildebrand et al. (Hrsg.), Daten- und Informationsqualität, https://doi.org/10.1007/978-3-658-30991-6}

Leider haben sich trotz sorgfältiger Prüfung Fehler eingeschlichen, die uns erst nach Drucklegung aufgefallen sind. Die nachfolgenden Korrekturen wurden jetzt ausgeführt. Im Original fehlten die Referenzen im Kapitel 21. Sie wurden nun an dieser Stelle eingefügt.

Apel, D., Behme, W., Eberlein, R., Merighi, C., Datenqualität erfolgreich steuern, München 2009

Baškarada, S., Information Quality Management Capability Maturity Model, Wiesbaden 2009

Batini, C., Scannapieco, M., Data Quality, Heidelberg 2006

Bleicher, K., Organisation. Strategien - Strukturen - Kulturen, Wiesbaden 1991

Chapman, P., Clinton, J., Kerber, R., Khabaza, T., Reinartz, T., Shearer, C., Wirth, R., CRISP-DM 1.0, Step-by-step data mining guide, 2000

Chrissis, M. B., Konrad, M., Shrum, S., CMMI for Development, 3.A, CMMI-DEV, Version 1.3, Boston 2011

Christ, O., Content-Management in der Praxis, Heidelberg 2003

Cohn, M., User Stories Applied, Boston 2004

Daconta M. C., u. a., The Semantic Web Guide, Wiley 2003

Date, C., Database Systems, 8.A, Boston 2004

Ebel, N., ITIL V3 Basiszertifizierung, Boston 2008

El Emam, K., Drouin, J. N., Melo, W., SPICE, Los Alamitos 1999

Die aktualisierte Version des Kapitels finden Sie unter:

https://doi.org/10.1007/978-3-658-30991-6_21 
Fowler, H., Higginson, T., Miller, L., Data Warehouse Maturity Assessment Service, Teradata 2006

Hanker, J., Die strategische Bedeutung der Informatik für Organisationen, Wiesbaden 1990

Hichert, R., Faisst, J., Gefüllt Gerahmt Schraffiert, München 2019

Hildebrand, K., Hinrichs, H., Gebauer, M., Mielke, M., Daten- und Informationsqualität, 4.A, Heidelberg 2018

Höhn, R., Höppner S., Das V-Modell XT, Heidelberg 2008

Höhn, R., Business Intelligence, in Breitbart G., Handbuch IT-Management, Augsburg 2017

Höhn, R., Integrata Seminarunterlage 2001-v2018-12 Vorgehensmodelle, Stuttgart 2018

Höhn, R., Integrata Seminarunterlage 2010-v2019-11 Anforderungsanalyse, Stuttgart 2019

Höhn, R., Integrata Seminarunterlage 5688-v2020-05 Datenqualitätsmanagement, Stuttgart 2020

IBM SPSS Modeler, CRISP-DM-Handbuch, 2012

Jung, R., Winter R., Data Warehousing Strategie, Heidelberg 2000

Juran, J. M., Juran's Quality Handbook, New York 2000

Kamsties E., Surfacing Ambiguity in Natural Language Requirements, Fraunhofer-Institut für Experimentelles Software Engineering 2001

Kemper A., Eickler A., Datenbanksysteme, Oldenbourg Verlag 2014

Kneuper, R., CMMI, Verbesserung von Software- und System-Entwicklungsprozessen mit Capability

Kosiol, E., Organisation der Unternehmung, Wiesbaden 1962

Krüger, W., Pfeiffer, P., Strategische Ausrichtung, organisatorische Gestaltung und Auswirkungen des Informationsmanagements, Information Management 2/88 S.6-15

Leser, U., Naumann, F., Informationsintegration, Heidelberg 2007

Maturity Model Integration, 3.A. Heidelberg 2007

Mathes, D., Enterprise Architecture Frameworks - Kompendium, Heidelberg 2011

McGilvray, D., Executing Data Quality Projects: Ten Steps to Quality Data, San Francisco 2008

Mosley, M., Bracket, M., Earley S., DAMA Guide to the Data Management Body of Knowledge, Bradley Beach 2010

Müller-Stewens, G., Lechner, C., Strategisches Management, 4.A, Stuttgart 2011

Munzner, T., Visualization Analysis \& Design, Boca Raton 2015

Naumann, F., Quality-Driven Query Answering for Integrated Information Systems, Heidelberg 2002

Nordsieck, F., Betriebsorganisation, Textband, 2.A, Stuttgart 1972

North K., Wissensorientierte Unternehmensführung, Gabler 2016

Österle, H., Business Engineering, Band 1 Entwurfstechniken, Heidelberg 1995

Österle, H., Winter, R., Business Engineering, Heidelberg 2003

Otto, B., Österle, H., Corporate Data Quality, Heidelberg 2016 
Pfeiffer, P., Technologische Grundlagen, Strategie und Organisation Gestaltung des Informationsmanagements, Berlin 1990

Porter, M. E., Wettbewerbsstrategie, Frankfurt 1988

Porter, M. E., Wettbewerbsvorteile, Frankfurt 1986

Probst, G., Raub, S., Romhardt, K., Wissen Managen, Wiesbaden 1998

Redman, T. C., Data Quality Management \& Technology, New York 1992

Riempp, G., Integrierte Wissensmanagementsysteme, Heidelberg 2004

Robertson S., Robertson J., Mastering the Requirements Process, Addison-Wesley Harlow 2012

Rupp, C., SOPHISTen, Requirements-Engineering und -Management, 6.A, München 2014

Sadiq, S., Eds., Handbook of Data Quality, Heidelberg 2013

Schreyögg, G., Conrad P., Managementforschung 6, Wissensmanagement, Berlin 1996

Schulte-Zurhausen, M., Organisation, 5.A, München 2011

Seidlmeier, H., Prozessmodellierung mit ARIS, 4.A, Heidelberg 2015

Stapelkamp, T., Informationsvisualisierung, Heidelberg 2013

Staehle, W. H., Conrad, P., Sydow J., Management, München 1999

Thome, G., Solbach, W., Grundlagen und Modelle des Information Lifecycle Management, Heidelberg 2007

Töpfer, A., Six Sigma, Heidelberg 2003

Ward, J., Peppard, J., The Strategic Management of Information Systems, 4.A, Chichester 2016

Weill, P., Ross J. W., IT Governance, Boston 2004

Welge, M. K., Al-Laham, A, Strategisches Management, 6.A, Heidelberg 2012

Zachmann, J. A., A Framework for Information Systems Architecture, IBM Systems Journal 26(3), 1989

Zangemeister, C., Nutzwertanalyse in der Systemtechnik, München 1976

Zollondz, H.-D., Grundlagen Qualitätsmanagement, München 2011

\section{Technische Normen und Standards}

CMMI for Acquisition Version 1.3, CMMI-ACQ, Software Engineering Institute, Carnegie Mellon University 2010

CMMI for Development Version 1.3, CMMI-ACQ, Software Engineering Institute, Carnegie Mellon University 2010

CMMI for Services Version 1.3, CMMI-ACQ, Software Engineering Institute, Carnegie Mellon University 2010

CMMI Development v2,0, CMMI Institute 2018

COBIT 5, Enabling Processes, ISACA 2012

COBIT 5, Process Assessment Model (PAM), ISACA 2013

COBIT 2019 Framework: Governance and Management Objectives, ISACA 2018,

COBIT 2019 Framework: Introduction and Methodology, ISACA 2018, 
DIN 66270:1998 Informationstechnik - Bewertung von Softwaredokumenten - Qualitätsmerkmale

DIN EN ISO 9000:2005 Qualitätsmanagementsysteme - Grundlagen und Begriffe

DIN EN ISO 9001:2000 Qualitätsmanagementsysteme - Anforderungen

El Emam, K., Drouin, J. N., Melo, W., SPICE, Los Alamitos 1999

FEAF, Federal Cio Council, Practical Guide to Federal Enterprise Architecture, www. cio.gov,

Fowler, H., Higginson, T., Miller, L., Data Warehouse Maturity Assessment Service, Teradata 2006

ISO 8000-1:2011 Data quality - Part 1: Overview

ISO 8000-8:2015 Data quality - Part 8: Information and data quality: Concepts and measuring

ISO 8000-61:2016 Data quality - Part 61: Data quality management: Process reference model

ISO 8000-62:2018 Data quality - Part 62: Data quality management: Organizational process maturity assessment: Application of standards relating to process assessment

ISO 8000-63:2019 Data quality - Part 63: Data quality management: process measurement

ISO 8000-130:2016 Data quality - Part 130: Master Data: Exchange of characteristic data: Accuracy

ISO 8000-140:2016 Data quality - Part 140: Master Data: Exchange of characteristic data: Completeness

ISO 8000-150:2011 Data quality - Part 150: Master Data: Quality management framework ISO 9001 Quality management systems - Requirements

ISO/IEC 12207:2017 Systems and software engineering - Software life cycle processes

ISO/IEC 15504-5:2012 Information technology - Process assessment - Part 5: An exemplar software life cycle process assessment model

ISO/IEC 15504-6:2013 Information technology - Process assessment - Part 6: An exemplar system life cycle process assessment model

ISO/IEC 15504-8:2012 Information technology - Process assessment - Part 8: An exemplar process assessment model for IT service management

ISO/IEC/IEEE 15288:2015 Systems and software engineering -System life cycle processes

ISO/IEC 15289:2006 Systems and software engineering - Content of systems and software life cycle process information products

ISO 19501:2012, Information technology - Open distriputed processing - Unified Modelling Language (UML)

ISO 19510:2013, Information technology - Open distriputed processing - Business Process Modelling \& Notation (BPM\&N)

ISO 19759:2005, Software engineering - Guide tot he Software Engineering Body of Knowledge (SWEBOK)

ISO/IEC 20000-1:2013, Information technology - Service Management - Part 1: General system Requirements 
ISO/IEC 25010:2011, Systems and software engineering - Systems and software Quality Requirements and Evaluation (SQuaRE) - Systems and software Quality models

ISO/IEC 25030:2007, Systems and software engineering - Software product Quality Requirements and Evaluation (SQuaRE) -Quality requirements

ISO/IEC 27001:2013, Information technology - Security technics - Information security management systems - Requirements

ISO/IEC/IEEE 29119-1:2013, Systems and software engineering - Software testing Part 1: Concepts and definitions

ISO/IEC/IEEE 29119-2:2013, Systems and software engineering - Software testing Part 2: Test processes

ISO/IEC/IEEE 29119-3:2013, Systems and software engineering - Software testing Part 3: Test documentation

ISO/IEC/IEEE 29119-4:2015, Systems and software engineering - Software testing Part 4: Test Techniques

ISO/IEC/IEEE 29148:2011(E), Systems and software engineering - Life cycle processes - Requirements engineering

ISO 31000:2009 Risk management - Principles and guidelines

ISO/IEC 31010:2009 Risk management - Risk assessment techniques

ISO/IEC 33002:2015 Information technology - Process assessment - Requirements for performing an assessment

ISO/IEC 33004:2015 Information technology - Process assessment - Requirements for process reference, process assessment and maturity models

ISO/IEC 33010:2015 Information technology - Process assessment - Process measurement - Guide on performing assessments

ISO/IEC 33014:2015 Information technology - Process assessment - Guide for process improvement

ISO/IEC 33020:2015 Information technology - Process assessment - Process Measurement framework for assessment of process capability and capability determination

ISO/IEC 33063:2015, Information technology - Process assessment - Process assessment model for software testing

ISO/IEC 38500:2015 ISO/IEC Information technology - Governance of IT for the organization

ISO/IEC TS 38502:2015 ISO/IEC Information technology - Governance of IT for the organization - Implementation guide

ISO/IEC TR 38502:2014 ISO/IEC Information technology - Governance of IT for the organization - framework and model

ISO/IEC/IEEE 42010:2011, Systems and software engineering -Architecture description

TOGAF 9.1 -The Book, The Open Group Architecture Framework, 2009, www. opengroup.or

V-Modell XT, www.cio-bund.der 